\title{
Impacto dos medicamentos nas intoxicações humanas no Brasil
}

\author{
Drug impact on human poisoning in Brazil
}

Maria Élide Bortoletto 1

Rosany Bochner 1

1 Sistema Nacional de Informações Tóxico-Farmacológicas, Centro de Informação Científica e Tecnológica, Fundação Oswaldo Cruz. Av. Brasil 4365, Prédio Biblioteca de Manguinhos, sala 218, Rio de Janeiro, $R J$ 21045-900, Brasil. melide@fiocruz.br rosany@fiocruz.br

\begin{abstract}
This article analyzes the role of pharmaceuticals in human poisoning in Brazil from 1993 to 1996, based on data from the National Network of Poison Control Centers. Results are presented according to cause, age group, sex, and evolution. The paper discusses measures aimed at preventing this serious public health problem, which primarily impacts children under five years of age in accidents caused by inappropriate use of medicines. The authors also analyze the association between female gender and deaths caused by intentional intake of drugs.
\end{abstract}

Key words Poisoning; Poison Control Centers; Drugs; Information Systems

Resumo O presente trabalho analisa a participação dos medicamentos nas intoxicações humanas no Brasil, no período de 1993 a 1996, com base nos dados registrados pela Rede Nacional de Centros de Controle de Intoxicações. Os resultados são apresentados segundo causa determinante, faixa etária, sexo e evolução. O trabalho discute e apresenta algumas sugestões de medidas visando a prevenção desse grave problema de saúde pública, em especial aqueles acidentes ocorridos em crianças menores de cinco anos, que constituíram o principal grupo envolvido com o consumo inadequado de medicamentos. O artigo também discute a participação significativa do sexo feminino nos óbitos provocados por ingestão intencional de medicamentos.

Palavras-chave Envenenamento; Centros de Controle de Intoxicações; Medicamentos; Sistemas de Informação 


\section{Introdução}

De 1993 a 1996, foram registrados no Brasil, pelo Sistema Nacional de Informações TóxicoFarmacológicas (SINITOX), 217.512 casos de intoxicação humana, com um total de 1.483 óbitos. Nesse período, os medicamentos se destacaram entre os agentes tóxicos, contribuindo com $27 \%$ dos casos registrados pela Rede de Centros de Controle de Intoxicações e ocupando o primeiro lugar nas estatísticas relativas a esses eventos. No conjunto dos 13 agentes tóxicos considerados pelo SINITOX, os medicamentos responderam por $62 \%$ das tentativas de suicídio registradas no período. Neste, a participação do sexo feminino nos casos registrados de intoxicação por medicamentos foi bastante expressiva: dos 57.748 casos, $63 \%$ eram mulheres. Do total de 101.396 casos de intoxicação, por todos os agentes, ocorridos com o sexo feminino, $36 \%$ foram causados por medicamentos e $20 \%$ por animais peçonhentos. As faixas etárias mais vulneráveis às intoxicações medicamentosas foram as de menores de cinco anos, com $33 \%$ dos casos, a de 20 a 29 anos, com $19 \%$, e a de 15 a 19 anos, com 13\%, contribuindo, juntas, com $65 \%$ do total dos casos. Dos 1.483 óbitos registrados no mesmo período, os medicamentos foram responsáveis por $18 \%$ dos óbitos, ocupando o segundo lugar. Os agrotóxicos apresentaram a maior letalidade, respondendo por $36 \%$ do total de óbitos registrados no período em análise, o que correspondeu a 536 casos fatais. O destaque observado no desempenho do medicamento enquanto agente tóxico no Brasil motivou a realização do presente trabalho.

O propósito do presente artigo é contribuir para a análise do impacto dos medicamentos nas intoxicações em seres humanos no Brasil, com base na avaliação do peso de sua participação no conjunto das intoxicações registradas no país, pela Rede SINITOX, no período de 1993 a 1996.

\section{Rede SINITOX}

O Sistema Nacional de Informações TóxicoFarmacológicas (SINITOX), criado em 1980 pelo Ministério da Saúde (MS), com sede na Fundação Oswaldo Cruz (FIOCRUZ), tem como principal atividade coordenar o processo de coleta, compilação, análise e divulgação dos casos de intoxicação humana registrados no país, pela Rede Nacional de Centros de Controle de Intoxicações, comumente denominada Rede SINITOX.
Nos anos de 1993 e 1994, a Rede SINITOX era formada por 28 centros de controle de intoxicações, localizados em 15 Estados, concentrados, até então, nas regiões Sudeste, Sul e Centro-Oeste. A partir de 1995, houve uma expansão da rede de centros nas regiões Norte e Nordeste. Em 1998, o país totalizava 32 centros, localizados em 17 dos 26 Estados. A Região Norte, com sete Estados, possui dois centros, um no Estado do Amazonas e outro no Pará. A Região Nordeste, com nove Estados, dispõe de centros em cinco Estados, sendo dois no Ceará, um no Rio Grande do Norte, dois na Paraíba, um em Pernambuco e um na Bahia. Na Região Sudeste, está localizado o maior número de centros: 11 no Estado de São Paulo, dois no Rio de Janeiro, um em Minas Gerais e um no Espírito Santo. Na Região Sul, estão em funcionamento cinco centros, dos quais três estão localizados no Estado do Paraná, um em Santa Catarina e um no Rio Grande do Sul. Na Região Centro-Oeste, estão em atividade três centros, um em Mato Grosso, um em Goiás e um em Mato Grosso do Sul.

Considerando o ano de 1996, a Região Norte possuía 0,9 centros por dez milhões de habitantes; a Região Nordeste, 1,3; a Região Sudeste, 2,2; a Região Sul, 2,1 e a Região Centro-Oeste, 2,9. O Brasil contava, naquele ano, com 1,9 centros por dez milhões de habitantes. Para a construção destes índices, utilizamos como população base a de 1996, estimada pelo Instituto Brasileiro de Geografia e Estatística (IBGE, 1998) em 156.803.232 habitantes, e o número de Centros de Controle de Intoxicações existentes naquele ano: 30 centros.

Os Estados Unidos possuíam, naquele mesmo ano de 1996, 67 centros e uma população estimada de 232.300.000 habitantes, o que representava 2,9 centros por dez milhões de habitantes (Litovitz et al., 1997).

Os Centros de Controle de Intoxicações funcionam em regime de plantão permanente, todos os dias do ano. Sua principal atividade é a prestação de informações aos profissionais de saúde, às instituições hospitalares e à população leiga, por plantonistas supervisionados pelo corpo técnico do centro, através de telefone, fax, e-mail. Nos atendimentos de emergência, os centros utilizam, como material básico de consulta, Monografias em Toxicologia de Urgência que compõem um banco de dados toxicológicos (CIT-RS/FIOCRUZ/ATOX, 1997). O banco é composto de trezentas monografias técnicas, envolvendo cinco grupos principais: medicamentos, pesticidas, produtos químicos de uso doméstico e industrial, plantas tóxicas e animais peçonhentos. As monografias com- 
preendem sumário, usos, sinônimos, fórmulas, descrição, mecanismos de ação, cinética, toxicologia, interações medicamentosas, efeitos clínicos, tratamento, análises laboratoriais, bibliografia.

A base de dados do SINITOX, criada em 1980 pelo Centro de Informação Toxicológica do Rio Grande do Sul, foi elaborada com adaptações à realidade brasileira, seguindo o modelo norte-americano da American Association of Poison Control Centers - AAPCC. Assim, os formulários preenchidos pela Rede de Centros de Controle de Intoxicações e enviados ao SINITOX contemplam os dados, segundo 13 categorias de agentes tóxicos: medicamentos, animais peçonhentos, animais não-peçonhentos, produtos químicos industriais, pesticidas agropecuários, pesticidas domésticos, raticidas, domissanitários, produtos de toalete, plantas, intoxicação por alimentos, outros produtos e não determinado.

Em 1997, uma comissão composta por representantes da Rede de Centros de Controle de Intoxicações, da Secretaria de Vigilância Sanitária e coordenada pela Fundação Oswaldo Cruz elaborou, a partir dos modelos utilizados pelos centros, uma ficha de notificação e de atendimento, objetivando buscar uma padronização de critérios e definições para orientar o processo de coleta de dados em todo o país. O novo modelo, ainda em fase de teste, que deverá ser implantado na Rede SINITOX a partir de 1999, contempla quatro novas categorias de agentes tóxicos (produtos veterinários, metais, drogas de abuso, outros animais venenosos), além de classificar os animais peçonhentos em serpentes, aranhas e escorpiões (Bortoletto et al., 1997).

\section{Metodologia}

As estatísticas divulgadas pelo SINITOX não contemplam a totalidade dos casos de intoxicação verificados no Brasil, porque, além do número de centros ser insuficiente para cobrir toda a extensão territorial do país - nove Estados e o Distrito Federal não dispõem de centros -, a notificação pelas vítimas ou seus familiares também é espontânea, ocorrendo, na maior parte das vezes, com o objetivo de obter informação sobre como proceder e onde buscar atendimento. Na maioria dos casos de intoxicação, o atendimento é buscado diretamente na rede de serviços de saúde, sem que haja registro junto aos centros (Marques et al., 1993). Tornar a notificação compulsória e implantar centros em todos os Estados são medidas que irão contribuir para diminuir o sub-registro desses eventos em nosso país.

O envio dos dados ao SINITOX, pelos Centros de Controle de Intoxicações, não é compulsório - os dados divulgados pelo SINITOX referem-se somente aos centros que enviam espontaneamente suas informações - além de ocorrer de forma descontínua, como pode ser observado na Tabela 1, dificultando a análise das tendências ao longo do período em estudo e impedindo a determinação do índice real de crescimento anual das intoxicações humanas. Dessa forma, a presente análise foi realizada com base na totalidade dos casos de intoxicação por medicamentos registrados por 29 dos trinta centros em atividade no país em 1996, que enviaram, no mínimo, dados referentes a um dos anos do período analisado.

A base de dados do SINITOX, ainda não informatizada, é composta por sete tabelas, seis referentes aos casos notificados e uma aos óbitos. Os casos registrados de intoxicação humana são distribuídos por treze agentes tóxicos combinados com cada uma das variáveis: causa determinante, faixa etária, sexo, zona de ocorrência e evolução. Por esta razão, os casos são analisados separadamente para cada uma das variáveis acima mencionadas.

Como os óbitos são apresentados, caso a caso, em uma única tabela, que informa o agente tóxico, a causa determinante, a faixa etária e o sexo, foi possível realizar uma análise baseada no conjunto dessas variáveis e não separadamente, como ocorreu com os casos notificados.

Os casos apresentados estão publicados nas estatísticas anuais do SINITOX e se referem aos anos de 1993, 1994, 1995 (dados revisados) e 1996 (FIOCRUZ/CICT/SINITOX, 1995, 1996, 1997, 1998).

Apesar dos fatores limitantes e condicionantes apontados em estudos anteriores (Bortoletto, 1990; Marques et al., 1993), consideramos que a importância do SINITOX resulta do fato de os centros de controle de intoxicações serem, no Brasil, as únicas fontes de registro de intoxicações e envenenamentos em seres humanos que classificam os casos registrados segundo o tipo de agente tóxico envolvido.

\section{Resultados e Discussão}

Na Tabela 1, são apresentados os casos e os óbitos por medicamentos, registrados para cada um dos centros de controle de intoxicações. A irregularidade no envio dos dados pode ser constatada nos centros localizados na Região Sudeste. 
Tabela 1

Casos de intoxicação humana por medicamentos e óbitos, segundo região do país e centro de controle. Brasil, 1993 a 1996.

\begin{tabular}{|c|c|c|c|c|c|c|c|c|c|c|}
\hline \multirow[t]{2}{*}{ Regiões/Centros } & \multicolumn{2}{|c|}{1993} & \multicolumn{2}{|c|}{1994} & \multicolumn{2}{|c|}{1995} & \multicolumn{2}{|c|}{1996} & \multicolumn{2}{|c|}{ Total no período } \\
\hline & Casos & Óbitos & Casos & Óbitos & Casos & Óbitos & Casos & Óbitos & Casos & Óbitos \\
\hline Norte &.. & .. & .. & .. & 16 & - & 23 & - & 39 & - \\
\hline CIT/AM - Manaus &.. & .. & .. & .. & 16 & - & 23 & - & 39 & - \\
\hline Nordeste & 2126 & 24 & 2320 & 18 & 2291 & 6 & 2498 & 22 & 9235 & 70 \\
\hline CEATOX/CE - Fortaleza & 943 & 9 & 1059 & 4 & 1128 & 2 & 1233 & 14 & 4363 & 29 \\
\hline CEATOX/CE - Iguatu &.. & .. & .. & .. & .. & .. & 73 & 1 & 73 & 1 \\
\hline CIT/RN - Natal & 33 & - & 42 & - & 31 & - & 18 & - & 124 & - \\
\hline CEATOX/PB - João Pessoa & 7 & - & 18 & - & 24 & - & 19 & - & 68 & - \\
\hline CAT/PE - Recife & 376 & 3 & 268 & 3 & 130 & - & 139 & 2 & 913 & 8 \\
\hline CIAVE/BA - Salvador & 767 & 12 & 933 & 11 & 978 & 4 & 1016 & 5 & 3694 & 32 \\
\hline Sudeste & 5445 & 22 & 7262 & 26 & 7375 & 21 & 11198 & 38 & 31280 & 107 \\
\hline ST/MG - Belo Horizonte & 1857 & 6 & 1909 & 1 & 2291 & 6 & 2570 & 9 & 8627 & 22 \\
\hline CCl/ES - Vitória & 349 & 2 & 315 & 4 & $\cdots$ & $\cdots$ & $\cdots$ & $\ldots$ & 664 & 6 \\
\hline CIT/RJ - Rio de Janeiro & 1169 & 6 & 1069 & 13 & 825 & 2 & 1276 & 4 & 4339 & 25 \\
\hline CCl/RJ - Niterói & 376 & 5 & 177 & 1 & 358 & 2 & 417 & 1 & 1328 & 9 \\
\hline CCl/SP - São Paulo & $\ldots$ & $\ldots$ & $\ldots$ & $\ldots$ & $\ldots$ & $\cdots$ & 2268 & 3 & 2268 & 3 \\
\hline CEATOX/SP - São Paulo & $\ldots$ & $\ldots$ & 1045 & - & 1195 & 2 & 1363 & 1 & 3603 & 3 \\
\hline $\mathrm{CCl} / \mathrm{SP}$ - Campinas & $\ldots$ & $\ldots$ & 983 & 4 & 957 & 6 & 1603 & 15 & 3543 & 25 \\
\hline $\mathrm{CCl} / \mathrm{SP}$ - Ribeirão Preto & 573 & 1 & 569 & - & 601 & - & 568 & 2 & 2311 & 3 \\
\hline CEATOX/SP - Botucatu & 26 & - & 36 & - & 7 & - & 14 & - & 83 & - \\
\hline $\mathrm{CCl} / \mathrm{SP}$ - São José dos Campos & 315 & 1 & 388 & - & 356 & - & 362 & - & 1421 & 1 \\
\hline CEATOX/SP - São José do Rio Preto & 277 & - & 349 & 3 & 358 & 1 & 332 & 2 & 1316 & 6 \\
\hline $\mathrm{CCl} / \mathrm{SP}$ - Taubaté & 365 & 1 & 294 & - & 301 & - & 195 & - & 1155 & 1 \\
\hline CEATOX/SP - Marília & $\cdots$ & $\cdots$ & $\cdots$ & $\cdots$ & $\cdots$ & $\cdots$ & $\cdots$ & $\cdots$ & $\cdots$ & $\cdots$ \\
\hline CEATOX/SP - Presidente Prudente & 138 & - & 128 & - & 105 & 2 & 111 & 1 & 482 & 3 \\
\hline $\mathrm{CCl} / \mathrm{SP}$ - Santos & $\ldots$ & $\ldots$ & $\ldots$ & $\ldots$ & 21 & - & 119 & - & 140 & - \\
\hline Sul & 3204 & 13 & 3440 & 11 & 3665 & 19 & 3901 & 19 & 14210 & 62 \\
\hline CIT/PR - Curitiba & 230 & - & 166 & 1 & 93 & - & 79 & 3 & 568 & 4 \\
\hline CCl/PR - Londrina & 220 & 3 & 256 & - & 264 & 1 & 234 & 1 & 974 & 5 \\
\hline $\mathrm{CCl} / \mathrm{PR}$ - Maringá & 358 & - & 330 & - & 278 & - & 353 & 3 & 1319 & 3 \\
\hline CIT/SC - Florianópolis & 434 & 2 & 489 & 3 & 575 & 4 & 575 & 3 & 2073 & 12 \\
\hline CIT/RS - Porto Alegre & 1962 & 8 & 2199 & 7 & 2455 & 14 & 2660 & 9 & 9276 & 38 \\
\hline Centro-oeste & 630 & 10 & 802 & 2 & 769 & 6 & 783 & 9 & 2984 & 27 \\
\hline CIT/MS - Campo Grande & 275 & 6 & 471 & 2 & 370 & 4 & 417 & 8 & 1533 & 20 \\
\hline CIAVE/MT - Cuiabá & 257 & 2 & 263 & - & 278 & 1 & 223 & 1 & 1021 & 4 \\
\hline CIT/GO - Goiânia & 98 & 2 & 68 & - & 121 & 1 & 143 & - & 430 & 3 \\
\hline Total & 11405 & 69 & 13824 & 57 & 14116 & 52 & 18403 & 88 & 57748 & 266 \\
\hline
\end{tabular}

Fonte: Ministério da Saúde, Fundação Oswaldo Cruz/SINITOX.

Sinais convencionais utilizados:

- Dado numérico igual a zero não resultante de arrendondamento.

.. Não se aplica dado numérico.

.. Dado numérico não disponível. 
No período, registrou-se um crescimento de $17 \%$ ao ano dos casos notificados de intoxicações por medicamentos. Este aumento pode ser atribuído a uma maior participação dos centros - em 1993, 23 centros enviaram seus dados ao SINITOX e, em 1996, esta participação passou para 28 centros -, a uma melhoria na coleta e registro dos dados, a um aumento real das intoxicações por medicamentos, entre outros fatores.

Os medicamentos vêm, desde 1994, segundo as estatísticas divulgadas pelo SINITOX, ocupando o primeiro lugar no conjunto dos 13 agentes tóxicos considerados, respondendo, no período de 1993 a 1996, por aproximadamente $27 \%$ dos casos de intoxicação registrados no país (Tabela 2). Este agente tóxico vem preocupando há algum tempo as autoridades e profissionais de saúde de países como os Estados Unidos (Litovitz et al., 1996, 1997, 1998), Costa Rica (Quirós-Vega et al., 1997, 1998), Uruguai (Burger, 1997) e Portugal (Borges, 1995, $1996,1997,1998)$, pelo aumento do volume dos casos de intoxicação que este agente provoca, colocando-o em primeiro lugar nas estatísticas desses países.

Os óbitos por medicamentos ocuparam o segundo lugar, precedidos pelos óbitos causados pelos pesticidas agropecuários, que apresentaram a maior letalidade, ou seja, $3 \%$. Os medicamentos, com um número bastante supe- rior de casos em relação aos pesticidas (57.748 para 17.831), apresentaram uma letalidade de $0,46 \%$ (Tabela 2).

A Tabela 3 mostra os casos e os óbitos por causa determinante. Verificamos que os acidentes, no conjunto dos agentes tóxicos, ocuparam o primeiro lugar, com 131.495 casos, correspondendo a aproximadamente $60 \%$ dos casos registrados, no período de 1993 a 1996.

Este comportamento é comum para a maioria dos agentes, com exceção dos medicamentos e dos raticidas, que apresentaram como principal causa determinante o suicídio. Do total de 57.748 casos de intoxicações medicamentosas, 25.297 (44\%) foram registrados como tentativas de suicídio e 23.150 (40\%) acidentais, perfazendo juntas $84 \%$ dos casos (Tabela 3 ).

Com relação aos 266 óbitos por medicamentos, 148 deles (56\%) foram atribuídos à causa "suicídio", seguidos de "outras causas" e “acidental" (Tabelas 2 e 3).

Do total de 40.918 casos de intoxicação humana relacionados à causa suicídio, os medicamentos foram responsáveis por $62 \%$ deste total. Este dado chama a atenção e põe em destaque o problema das intoxicações por medicamentos, objeto de nosso estudo. Com relação a outras causas, os medicamentos foram responsáveis por $60 \%$ dos casos e, para a causa acidente, responderam por $18 \%$ dos 131.495 casos referentes a esta causa (Tabela 3 ).

Casos, óbitos e letalidade por agente tóxico. Brasil, 1993 a 1996.

\begin{tabular}{|c|c|c|c|c|c|}
\hline \multirow[t]{2}{*}{ Agentes } & \multicolumn{2}{|c|}{ Casos } & \multicolumn{2}{|c|}{ Óbitos } & \multirow{2}{*}{$\begin{array}{c}\text { Letalidade } \\
\%\end{array}$} \\
\hline & $n$ & $\%$ & $\mathrm{n}$ & $\%$ & \\
\hline Medicamentos & 57748 & 26,5 & 266 & 17,9 & 0,46 \\
\hline Animais peçonhentos & 53763 & 24,7 & 168 & 11,3 & 0,31 \\
\hline Animais não-peçonhentos & 17604 & 8,1 & 10 & 0,7 & 0,06 \\
\hline Produtos químicos industriais & 16784 & 7,7 & 120 & 8,1 & 0,71 \\
\hline Pesticidas agropecuários & 17831 & 8,2 & 536 & 36,1 & 3,01 \\
\hline Pesticidas domésticos & 6963 & 3,2 & 48 & 3,2 & 0,69 \\
\hline Raticidas & 5758 & 2,6 & 108 & 7,3 & 1,88 \\
\hline Domissanitários & 15841 & 7,3 & 38 & 2,6 & 0,24 \\
\hline Produtos de toalete & 1478 & 0,7 & - & - & - \\
\hline Plantas & 5864 & 2,7 & 31 & 2,1 & 0,53 \\
\hline Intoxicação por alimentos & 3341 & 1,5 & 11 & 0,7 & 0,33 \\
\hline Outros produtos & 10616 & 4,9 & 60 & 4,0 & 0,57 \\
\hline Não determinado & 3921 & 1,8 & 87 & 5,9 & 2,22 \\
\hline Total & 217512 & 100,0 & 1483 & 100,0 & 0,68 \\
\hline
\end{tabular}

Fonte: Ministério da Saúde, Fundação Oswaldo Cruz/SINITOX.

Sinal convencional utilizado:

- Dado numérico igual a zero não resultante de arrendondamento. 
Casos de intoxicação humana e óbitos por causa determinante. Brasil, 1993 a 1996.

\begin{tabular}{|c|c|c|c|c|c|c|c|c|c|c|c|c|}
\hline \multirow{3}{*}{ Agentes } & \multicolumn{12}{|c|}{ Causas } \\
\hline & \multicolumn{2}{|c|}{ Acidente } & \multicolumn{2}{|c|}{ Abuso } & \multicolumn{2}{|c|}{ Suicídio } & \multicolumn{2}{|c|}{ Profissional } & \multicolumn{2}{|c|}{ Outras } & \multicolumn{2}{|c|}{ Ignorada } \\
\hline & Casos & Óbitos & Casos & Óbitos & Casos & Óbitos & Casos & Óbitos & Casos & Óbitos & Casos & Óbitos \\
\hline Medicamentos & 23150 & 42 & 1842 & 1 & 25297 & 148 & 144 & 1 & 5787 & 43 & 1528 & 31 \\
\hline Animais Peçonhentos & 44771 & 140 & 2 & - & 4 & - & 7364 & 23 & 508 & 2 & 1114 & 3 \\
\hline Animais não-peçonhentos & 15839 & 10 & - & - & 4 & - & 1253 & - & 209 & - & 299 & - \\
\hline Produtos químicos industriais & 10514 & 23 & 637 & 12 & 1466 & 63 & 3478 & 6 & 362 & 6 & 327 & 10 \\
\hline Pesticidas agropecuários & 6696 & 50 & 42 & 2 & 5449 & 391 & 4915 & 36 & 338 & 8 & 391 & 49 \\
\hline Pesticidas domésticos & 3843 & 8 & 52 & - & 2530 & 31 & 200 & 2 & 188 & 2 & 150 & 5 \\
\hline Raticidas & 2526 & 14 & 9 & - & 2934 & 83 & 31 & - & 113 & 4 & 145 & 7 \\
\hline Domissanitários & 12195 & 9 & 50 & - & 2229 & 27 & 917 & 1 & 272 & 1 & 178 & - \\
\hline Produtos de toalete & 1318 & - & 5 & - & 60 & - & 13 & - & 62 & - & 20 & - \\
\hline Plantas & 4589 & 16 & 382 & 5 & 202 & - & 98 & - & 501 & 10 & 92 & - \\
\hline Intoxicação por alimentos & 2754 & 10 & 34 & - & 7 & - & 51 & - & 390 & - & 105 & 1 \\
\hline Outros produtos & 1670 & 9 & 6959 & 27 & 405 & 8 & 1039 & - & 319 & 10 & 224 & 6 \\
\hline Não determinado & 1630 & 9 & 73 & - & 331 & 22 & 123 & - & 547 & 7 & 1217 & 49 \\
\hline Total & 131495 & 340 & 10087 & 47 & 40918 & 773 & 19626 & 69 & 9596 & 93 & 5790 & 161 \\
\hline
\end{tabular}

Fonte: Ministério da Saúde, Fundação Oswaldo Cruz/SINITOX.

Sinal convencional utilizado:

- Dado numérico igual a zero não resultante de arrendondamento.

A distribuição dos casos, dos óbitos e da letalidade por medicamentos segundo faixa etária, no período em análise, constam da Tabela 4. Crianças menores de cinco anos são as maiores vítimas das intoxicações causadas por medicamentos, participando com $33 \%$ dos casos registrados em nosso país. Adultos, de 20 a 29 anos, constituem a segunda faixa etária mais vulnerável, com 19\% dos casos registrados, seguida pelas faixas de 15 a 19 anos (13\%) e de trinta a 39 anos (11\%).

Com relação aos óbitos, os adultos de vinte a 29 anos responderam por $22 \%$ das mortes por medicamentos, seguidos do grupo de 30 a 39 anos $(18 \%)$ e das crianças menores de cinco anos, $16 \%$ dos óbitos.

A letalidade, a partir da faixa de cinco a nove anos, vai crescendo com o aumento da idade até atingir a faixa de 60 a 69 anos. Crianças menores de cinco anos apresentam letalidade próxima à da faixa de 15 a 19 anos e a de adultos com 70 anos ou mais é um pouco inferior à da faixa de 60 a 69 anos, mas ainda superior à das demais faixas etárias (Tabela 4).

Verifica-se na Tabela 5 que, do total de casos e de óbitos registrados para o conjunto dos agentes tóxicos, houve uma predominância do sexo masculino, com $53 \%$ dos casos e $64 \%$ dos óbitos. O sexo feminino contribuiu com $47 \%$ dos casos e $35 \%$ dos óbitos. Os animais peçonhentos, os medicamentos, os pesticidas agro- pecuários e os produtos químicos industriais foram os principais agentes que causaram intoxicações em pessoas do sexo masculino. Já para o grupo feminino foram os medicamentos, os animais peçonhentos, os domissanitários, os animais não-peçonhentos e os pesticidas agropecuários.

Quando se analisam os óbitos, o agente pesticida agropecuário foi o que apresentou a maior letalidade, ocupando a primeira posição para ambos os sexos. Em segundo lugar, apareceram os animais peçonhentos, para o sexo masculino, e os medicamentos, para o sexo feminino. Em terceiro lugar, ocorre uma inversão dos agentes, os medicamentos para o sexo masculino e os animais peçonhentos para o sexo feminino.

A participação do sexo feminino é bastante expressiva quando se analisam as intoxicações por medicamentos. Verificou-se que, do total de 57.748 casos atribuídos àquele agente, mais da metade (63\%) correspondeu ao sexo feminino e, do total de 266 óbitos atribuídos aos medicamentos, 151 (57\%) ocorreram com o sexo feminino. Verifica-se, dessa forma, que, tanto em relação aos casos quanto aos óbitos, o sexo feminino apareceu como o grupo mais vulnerável. Contudo, a letalidade das intoxicações medicamentosas para o sexo masculino $(0,54 \%)$ é superior à apresentada pelo sexo feminino $(0,41 \%)$ (Tabela 5$)$. 
Casos, óbitos e letalidade de intoxicação por medicamentos segundo faixa etária. Brasil, 1993 a 1996.

\begin{tabular}{lrrrrr}
\hline Faixa etária & \multicolumn{2}{c}{ Casos } & \multicolumn{2}{c}{ Óbitos } & \multicolumn{2}{c}{ Letalidade } \\
& & & $n$ & $\%$ & $\%$ \\
\hline$<5$ & 19045 & 33,0 & 42 & 15,7 & 0,22 \\
5 a 9 & 3496 & 6,1 & 3 & 1,1 & 0,09 \\
10 a 14 & 3324 & 5,8 & 5 & 1,9 & 0,15 \\
15 a 19 & 7626 & 13,2 & 18 & 6,8 & 0,24 \\
20 a 29 & 10756 & 18,6 & 58 & 21,8 & 0,54 \\
30 a 39 & 6402 & 11,1 & 47 & 17,7 & 0,73 \\
40 a 49 & 3218 & 5,6 & 31 & 11,7 & 0,96 \\
50 a 59 & 1251 & 2,2 & 24 & 9,0 & 1,92 \\
60 a 69 & 594 & 1,0 & 16 & 6,0 & 2,69 \\
70 e + & 439 & 0,7 & 10 & 3,8 & 2,28 \\
Ignorada & 1597 & 2,8 & 12 & 4,5 & 0,75 \\
Total & 57748 & 100,0 & 266 & 100,0 & 0,46 \\
\hline
\end{tabular}

Fonte: Ministério da Saúde, Fundação Oswaldo Cruz/SINITOX.

Tabela 5

Casos de intoxicação humana e óbitos distribuídos por sexo. Brasil, 1993 a 1996.

\begin{tabular}{|c|c|c|c|c|c|c|c|c|}
\hline \multirow[t]{2}{*}{ Agentes } & \multicolumn{2}{|c|}{ Masculino } & \multicolumn{2}{|c|}{ Feminino } & \multicolumn{2}{|c|}{ Ignorado } & \multicolumn{2}{|c|}{ Total } \\
\hline & Casos & Óbitos & Casos & Óbitos & Casos & Óbitos & Casos & Óbitos \\
\hline Medicamentos & 20696 & 112 & 36475 & 151 & 577 & 3 & 57748 & 266 \\
\hline Animais peçonhentos & 32882 & 119 & 20733 & 48 & 148 & 1 & 53763 & 168 \\
\hline Animais não-peçonhentos & 9709 & 6 & 7835 & 4 & 60 & - & 17604 & 10 \\
\hline Produtos químicos industriais & 10406 & 85 & 6039 & 35 & 339 & - & 16784 & 120 \\
\hline Pesticidas agropecuários & 11563 & 376 & 6139 & 154 & 129 & 6 & 17831 & 536 \\
\hline Pesticidas domésticos & 3166 & 34 & 3726 & 14 & 71 & - & 6963 & 48 \\
\hline Raticidas & 2709 & 70 & 2988 & 38 & 61 & - & 5758 & 108 \\
\hline Domissanitários & 7783 & 22 & 7844 & 15 & 214 & 1 & 15841 & 38 \\
\hline Produtos de toalete & 677 & - & 785 & - & 16 & - & 1478 & - \\
\hline Plantas & 3043 & 14 & 2765 & 16 & 56 & 1 & 5864 & 31 \\
\hline Intoxicação por alimentos & 1636 & 7 & 1677 & 4 & 28 & - & 3341 & 11 \\
\hline Outros produtos & 8045 & 52 & 2503 & 8 & 68 & - & 10616 & 60 \\
\hline Não determinado & 1959 & 51 & 1887 & 33 & 75 & 3 & 3921 & 87 \\
\hline Total & 114274 & 948 & 101396 & 520 & 1842 & 15 & 217512 & 1483 \\
\hline
\end{tabular}

Fonte: Ministério da Saúde, Fundação Oswaldo Cruz/SINITOX.

Sinal convencional utilizado:

- Dado numérico igual a zero não resultante de arrendondamento. 
Outro fato que mostra um maior envolvimento do sexo feminino nas intoxicações medicamentosas pode ser verificado também na Tabela 5, onde $36 \%$ do total de 101.396 casos de intoxicação em pessoas do sexo feminino foram atribuídos aos medicamentos, enquanto que, para o sexo masculino, este percentual cai para a metade, ou seja, $18 \%$.

Do total de 520 óbitos registrados para o sexo feminino, $29 \%$ foram causados pelos medicamentos, enquanto que, para o sexo masculino, os medicamentos contribuíram com $12 \%$ dos óbitos.

Através da Tabela 6, podemos verificar a distribuição dos óbitos por medicamentos por faixa etária para cada uma das causas. Para acidente, $50 \%$ dos óbitos se concentraram em crianças menores de cinco anos. O suicídio surge como causa de óbito a partir da faixa etária de 10 a 14 anos, atingindo mais intensamente adultos de 20 a 29 anos, que apresentaram $29 \%$ dos óbitos por esta causa. A causa “outras" tem nas crianças menores de cinco anos a maior participação, $44 \%$. Adultos de vinte a 49 anos contribuíram com $58 \%$ dos óbitos de causa ignorada.

A maioria dos óbitos registrados em crianças menores de um ano, 63\%, estavam relacionados a outras causas, seguidos por acidente, com $32 \%$. Crianças de um a quatro anos apresentaram exatamente o inverso, $65 \%$ dos óbitos estavam relacionados a acidentes e $30 \%$ a outras causas. Este comportamento pode estar relacionado ao desenvolvimento psicomotor da criança (Scherz, 1970). Crianças de cinco a nove anos apresentaram apenas três óbitos. Para todas as faixas etárias compreendidas entre 10 e 69 anos, o suicídio constituiu a principal causa de óbito. Adultos com setenta anos e mais contribuíram com dez óbitos, "outras causas” foram responsáveis por $40 \%$ deste total e o suicídio por $30 \%$.

Dos 151 óbitos por medicamentos ocorridos com pessoas do sexo feminino, os adultos de 20 a 29 anos apresentaram a maior participação, 26\%, seguidos pela faixa de 30 a 39 (15\%). Para as pessoas do sexo masculino, ocorre uma inversão: dos 112 óbitos registrados, os adultos de 30 a 39 anos participaram com $21 \%$, seguidos pela faixa de 20 a 29 anos, com $16 \%$.

$\mathrm{Na}$ análise das causas determinantes, foi constatado que $64 \%$ dos óbitos acidentais e $62 \%$ dos suicídios ocorreram em pessoas do sexo feminino. A faixa etária de 20 a 29 anos apresentou o maior percentual de participação do sexo feminino nos óbitos por medicamentos, relacionados à causa suicídio, ou seja, $74 \%$.

\section{Conclusões}

O presente estudo mostrou que $60 \%$ das intoxicações causadas pelo conjunto dos 13 agentes tóxicos são acidentais e, portanto, evitáveis. Os medicamentos - a exemplo do que ocorre na maioria dos países desenvolvidos - passaram a ocupar a primeira posição, também em nosso país, no quadro dos agentes tóxicos que mais causam intoxicações em seres humanos. A análise dos casos de intoxicação provocados por medicamentos mostraram que $40 \%$ do total são acidentais e as maiores vítimas são as crianças menores de cinco anos. Sibert \& Routledge (1991) elaboraram um quadro, onde são apresentados os três principais grupos de substâncias que mais causam intoxicações acidentais em crianças, a saber: os medicamentos, os produtos domissanitários e as plantas.

Sibert (1975) associa o estresse familiar com a ocorrência de intoxicações acidentais com crianças. Como exemplos de estresse familiar, o autor considera: doença grave na família, perda de pais, avós ou irmão gêmeo no último mês, mãe grávida, mudança de casa nos últimos três meses, ausência de um dos pais (inclui separações e divórcios e também ausência temporária), pai desempregado, pai ou mãe ansiosos ou deprimidos a ponto de procurar um médico nos últimos três meses. Esta associação ao estresse familiar demanda estudos brasileiros. Outros fatores, entretanto, merecem ser considerados fora do âmbito da família.

No caso das intoxicações acidentais com crianças menores de cinco anos, compartilhamos e defendemos a adoção, em nosso país, da Embalagem Especial de Proteção à Criança EEPC - em medicamentos e produtos químicos de uso doméstico que apresentem potencial de risco à saúde (Brasil, 1994). Walton (1982) estima que, a partir de 1973, este tipo de embalagem preveniu cerca de duzentos mil casos de ingestão acidental em crianças menores de cinco anos, provocados por um grupo de 15 produtos regulamentados pela lei que implantou, nos Estados Unidos, em 1970, o uso de embalagem de proteção à criança em medicamentos e produtos químicos domésticos. $\mathrm{O}$ autor estima, ainda, que, nos últimos 20 anos, a taxa de mortalidade causada por intoxicações em crianças diminuiu de 2,0 por cem mil para 0,5 por cem mil. Rodgers (1996) concluiu, através de um modelo de regressão múltipla, que a embalagem especial de proteção à criança reduziu a taxa de mortalidade infantil causada por ingestão acidental de medicamentos, nos Estados Unidos, no período de 1974 a 1992. Sibert (1975) afirma que a única medida que tem se mostra- 
Distribuição dos óbitos por medicamentos segundo faixa etária, causa determinante e sexo. Brasil, 1993 a 1996.

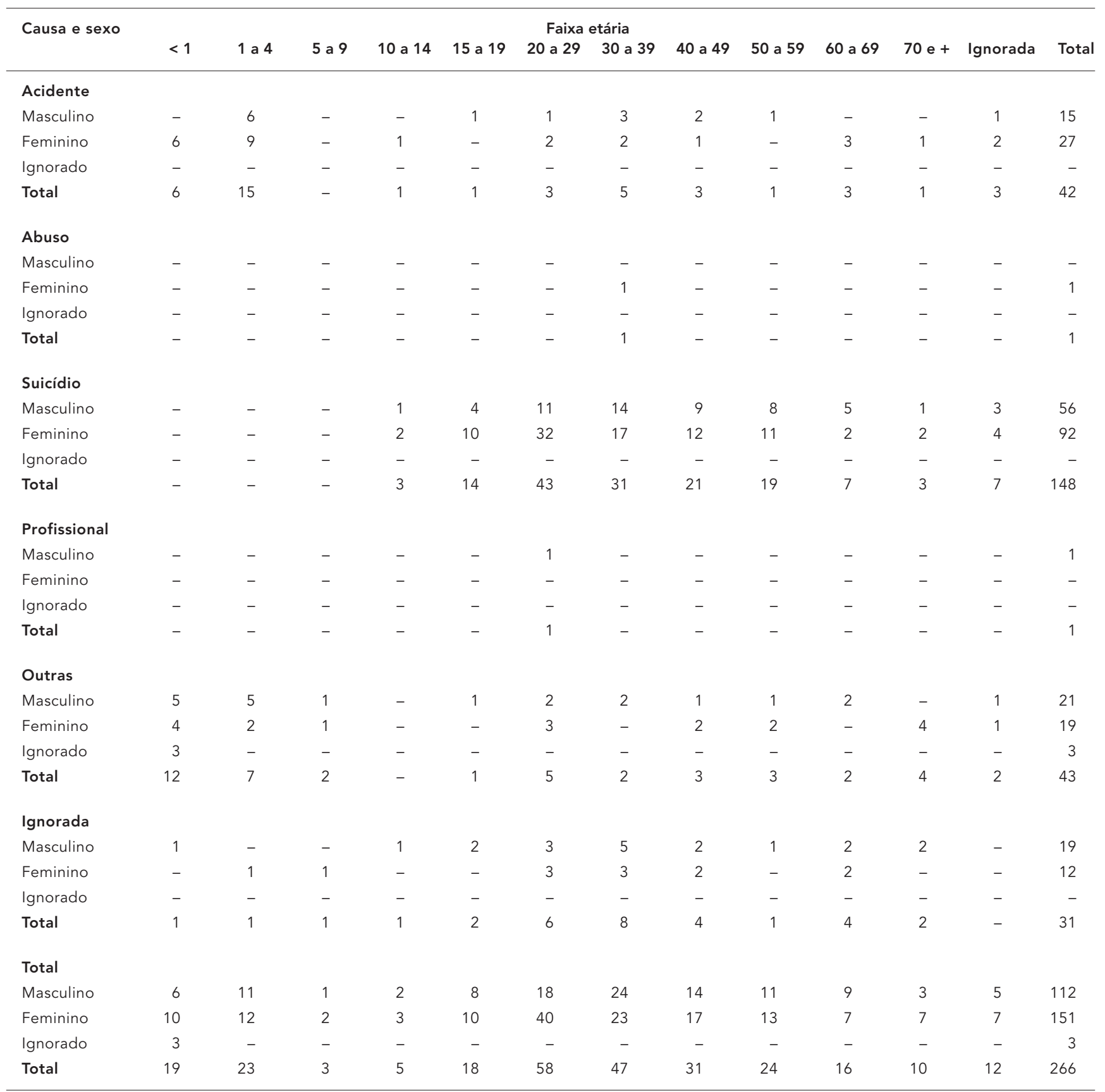

Fonte: Ministério da Saúde, Fundação Oswaldo Cruz/SINITOX.

Sinal convencional utilizado:

- Dado numérico igual a zero não resultante de arrendondamento. 
do eficiente na redução da incidência de intoxicações na infância é o uso de embalagem especial de proteção à criança. O estudo de Scherz (1970) mostrou uma redução de 149 para 17 no número de intoxicações em uma comunidade. Dos 17 casos, seis ocorreram com crianças que tinham, em média, quatro anos e três meses e que conseguiram abrir as embalagens de segurança. Nos outros 11 casos, ocorreu, de alguma forma, violação do princípio de segurança da embalagem, provavelmente, pelos pais.

Ainda, para este autor, as intoxicações acidentais em crianças compreendem uma complexa interação entre a criança, a substância tóxica e o ambiente, interação esta que deverá ser sempre considerada quando do desenvolvimento de programas de prevenção de acidentes com crianças.

A implementação de programas educacionais e de prevenção de intoxicações acidentais, nas escolas, nas creches, nas comunidades, associados à utilização de embalagens de proteção à criança, aos cuidados com a armazenagem de medicamentos e produtos químicos de uso doméstico, irá contribuir de forma significativa para a diminuição dos casos de intoxicação acidental por medicamentos com crianças.

Fatores como a utilização abusiva pelas indústrias de embalagens atraentes, medicamentos coloridos e adocicados, com sabor de frutas e formato de bichinhos, colaboram para o aumento das intoxicações acidentais com crianças.

Outra constatação da presente análise foi a expressiva participação do sexo feminino nas intoxicações por medicamentos, $63 \%$ dos casos e $57 \%$ dos óbitos ocorreram com mulheres.

Segundo Rozenfeld (1989), com exceção dos dados publicados por empresas voltadas para o marketing farmacêutico, não se conhece publicação alguma patrocinada por instituições públicas ou privadas que realizem, de forma sistemática, a coleta de dados sobre o consumo de medicamentos, que proporcionem o acompanhamento da evolução dos padrões de utilização ao longo do tempo. Segundo a autora, o único levantamento oficial, abrangente e detalhado sobre o consumo de medicamentos foi realizado em 1971, pela extinta Central de Medicamentos, do Ministério da Saúde.
A faixa etária em que mais ocorreram óbitos foi a de 20 a 29 anos, respondendo por 58 óbitos. Neste grupo, destaca-se a participação do sexo feminino com 40 óbitos, ou seja, $69 \%$ do total. Para Rozenfeld (1989), o padrão de consumo de medicamentos da população é caracterizado, em geral, por elevados níveis de uso de drogas sintomáticas e pela automedicação.

Os resultados do estudo de Arrais et al. (1997) sobre o perfil da automedicação no Brasil sugerem que a automedicação em nosso país é praticada principalmente por mulheres na faixa etária entre 16 e 45 anos.

Finalizando, o impacto dos medicamentos nas intoxicações humanas no Brasil, tendo em conta a avaliação do peso de sua participação no conjunto dos agentes tóxicos registrados pelo SINITOX, pode ser sintetizado da seguinte forma: - medicamento é o principal agente tóxico que causa intoxicação em seres humanos no país, ocupando o primeiro lugar, nas estatísticas do SINITOX, desde 1994;

- os benzodiazepínicos, antigripais, antidepressivos, anti-inflamatórios são as classes de medicamentos que mais causam intoxicações em nosso país;

- $44 \%$ do total das intoxicações medicamentosas foram classificadas como tentativas de suicídio e $40 \%$, como acidentes;

- do total registrado das tentativas de suicídio, $62 \%$ foram atribuídas aos medicamentos;

- o sexo feminino é o mais vulnerável - do total das intoxicações e do total dos óbitos atribuídos aos medicamentos, $63 \%$ e $57 \%$, respectivamente, ocorreram no sexo feminino;

- $36 \%$ do total das intoxicações verificadas no sexo feminino ocorreram com medicamentos;

- crianças menores de cinco anos (33\%) e adultos de 20 a 29 anos (19\%) constituíram as faixas etárias mais acometidas pelas intoxicações por medicamentos;

- as faixas etárias de 20 a 29 anos (58 óbitos), de 30 a 39 anos (47 óbitos) e menores de cinco anos (42 óbitos) constituíram os grupos mais vulneráveis, contribuindo, juntos, com 55\% dos óbitos registrados por medicamentos;

- na faixa etária de 20 a 29 anos, a participação do sexo feminino foi bastante expressiva, contribuindo com $69 \%$ do total dos óbitos registrados neste grupo etário.

\section{Agradecimentos}

Agradecemos aos 29 Centros de Controle de Intoxicações que, ao enviarem seus dados, possibilitaram a realização deste estudo. 


\section{Referências}

ARRAIS, P. S. D.; COELHO, H. L. L.; BATISTA, M. C. D S.; CARVALHO, M. L.; RIGHI, R. E. \& ARNAU, J. M., 1997. Perfil da automedicação no Brasil. Revista de Saúde Pública, 31:71-77.

BORGES, A., 1995. Portugal 1994 Annual Report. Lisboa: Centro de Informação Antivenenos, Instituto Nacional de Emergência Médica, Ministério da Saúde.

BORGES, A., 1996. Portugal 1995 Annual Report. Lisboa: Centro de Informação Antivenenos, Instituto Nacional de Emergência Médica, Ministério da Saúde.

BORGES, A., 1997. Portugal 1996 Annual Report. Lisboa: Centro de Informação Antivenenos, Instituto Nacional de Emergência Médica, Ministério da Saúde.

BORGES, A., 1998. Portugal 1997 Annual Report. Lisboa: Centro de Informação Antivenenos, Instituto Nacional de Emergência Médica, Ministério da Saúde.

BORTOletTO, M. E., 1990. Tóxicos, Civilização e Saúde. Contribuição à Análise dos Sistemas de Informações Tóxico-Farmacológicas no Brasil. Rio de Janeiro : FIOCRUZ.

BORTOLETTO, M. E.; NICOLELLA, A.; FERREIRA, E. M.; WONG, A.; FRUCHTENGARTEN, L.; PINHEIRO, M. S. B.; ZAMBRONE, F.; ALONZO, H. G. A.; BENATTO, A.; EVANGELHISTA-FILHO, D.; MENDES, A.; BOCHNER, R. \& CHRISTENSEN, R. A., 1997. Manual de Preenchimento da Ficha de Notificação e de Atendimento - Centros de Assistência Toxicológica. Rio de Janeiro: Fundação Oswaldo Cruz.

BRASIL, 1994. Congresso Nacional, Câmara dos Deputados. Projeto de Lei no 4841, de 1994, de Fábio Feldman. Determina a utilização de embalagem especial de proteção à criança - EEPC - em medicamentos e produtos químicos de uso doméstico que apresentem potencial de risco à saúde. Brasília: Congresso Nacional.

BURGER, M., 1997. Informe de Actividades Año 1996. Montevideo: Centro de Información y Asesoramiento Toxicológico.

CIT-RS/FIOCRUZ/ATOX (Centro de Informação Toxicológica do Rio Grande do Sul/Fundação Oswaldo Cruz/Associação de Toxicologia Aplicada), 1997. Monografias em Toxicologia de Urgência, Vol. 1/6. Porto Alegre: CIT-RS/FIOCRUZ/ATOX.

FIOCRUZ/CICT/SINITOX (Fundação Oswaldo Cruz/ Centro de Informação Científica e Tecnológica/ Sistema Nacional de Informações Tóxico-Farmacológicas), 1998. Estatística Anual de Casos de Intoxicação e Envenenamento. Brasil, 1996. Rio de Janeiro: FIOCRUZ/CICT/SINITOX.

FIOCRUZ/CICT/SINITOX (Fundação Oswaldo Cruz/ Centro de Informação Científica e Tecnológica/ Sistema Nacional de Informações Tóxico-Farmacológicas), 1997. Estatística Anual de Casos de Intoxicação e Envenenamento. Brasil, 1995. Rio de Janeiro: FIOCRUZ/CICT/SINITOX

FIOCRUZ/CICT/SINITOX (Fundação Oswaldo Cruz/ Centro de Informação Científica e Tecnológica/ Sistema Nacional de Informações Tóxico-Farmacológicas), 1996. Estatística Anual de Casos de In- toxicação e Envenenamento. Brasil, 1994. Rio de Janeiro: FIOCRUZ/CICT/SINITOX.

FIOCRUZ/CICT/SINITOX (Fundação Oswaldo Cruz/ Centro de Informação Científica e Tecnológica/ Sistema Nacional de Informações Tóxico-Farmacológicas), 1995. Estatística Anual de Casos de Intoxicação e Envenenamento. Brasil, 1993. Rio de Janeiro: FIOCRUZ/CICT/SINITOX.

IBGE (Fundação Instituto Brasileiro de Geografia e Estatística), 1998. Contagem da População - 1996. 12 de novembro de 1998 <http://www.ibge.gov. br/ informacoes/censo96/defdpe/dr_cont_96.html>.

LITOVITZ, T. L.; SCHWARTZ, W. K.; DYER, K. S.; SHANNON, M.; LEE, S. \& POWERS, M., 1998. 1997 Annual Report of the American Association of Poison Control Centers Toxic Exposure Surveillance System. American Jounal of Emergency Medicine, 16:443-497.

LITOVITZ, T. L.; SMILKSTEIN, M.; FELBERG, L.; SCHWARTZ, W. K., BERLIN, R. \& MORGAN, J. L., 1997. 1996 Annual Report of the American Association of Poison Control Centers Toxic Exposure Surveillance System. American Jounal of Emergency Medicine, 15:447-500.

LITOVITZ, T. L.; FELBERG, L.; WHITE, S. \& SCHWARTZ, W. K., 1996. 1995 Annual Report of the American Association of Poison Control Centers Toxic Exposure Surveillance System. American Jounal of Emergency Medicine, 14:487-537.

MARQUES, M. B.; BORTOLETTO, M. E.; FREITAS, C. M.; BEZERRA, M. C. C. \& SANTANA, R. A. L., 1993. Intoxicações e envenenamentos acidentais no Brasil: Análise epidemiológica dos casos registrados pelo Sistema Nacional de Informações Tóxico-Farmacológicas - SINITOX. Informe Epidemiológico do SUS, 4:59-93.

QUIROS-VEGA, D.; SALAS-HERRERA, A. E. \& EARLE, Y. L., 1997. Informe Anual de Intoxicaciones de 1996. San José: Centro Nacional de Controle de Intoxicaciones de Costa Rica.

QUIROS-VEGA, D.; SALAS-HERRERA, A. E. \& EARLE, Y. L., 1998. Informe Anual de Intoxicaciones de 1997. San José: Centro Nacional de Controle de Intoxicaciones de Costa Rica.

RODGERS, G. B., 1996. The safety effects of child-resistant packaging for oral prescription drugs: Two decades of experience. Journal of the American Medical Association, 275:1661-1665.

ROZENFELD, S., 1989. O uso de medicamentos no Brasil. In: Epidemiologia do Medicamento. Princípios Gerais (J. R. Laporte, G. Tognoni \& S. Rozenfeld, org.), pp. 21-41, São Paulo: Editora Hucitec/ Rio de Janeiro: Abrasco.

SCHERZ, R. G., 1970. Prevention of Childhood Poisoning. Pediatric Clinics of North America, 17:713-727.

SIBERT, J. R., 1975. Stress in families of children who have ingested poisons. British Medical Journal, 3:87-89.

SIBERT, J. R. \& ROUTLEDGE, P. A., 1991. Accidental poisoning in children: Can we admit fewer children with safety? Archives of Disease in Childhood, 66:263-266.

WALTON, W. W., 1982. An evaluation of the poison prevention packaging act. Pediatrics, 69:363-370. 